Wir sind entschlossen, das Auskunftsbuch für die chemische Industrie nach dem Plane, den alle Kritiken und Zuschriften für einen guten und richtigen erklärt haben, weiter auszubauen und - unterstützt von geeigneten Mitarbeitern - stetig an seiner Vervollkommnung zu arbeiten. Wir hoffen, dass das chemische Auskunftsbuch, dessen niedriger Bezugspreis eine Anschaffung jedem ermöglicht, sich auch weiter seine schnell erworbene Beliebtheit bewahren, dass es sich zu den vielen alten zahlreiche neue Freunde gewinnen wird, dass es sich in den Räumen der Fabriken, im Laboratorium und in der Studierstube einen festen Platz erringen, dass es den schon errungenen sich erhalten, dass es als chemisches Jahrbuch in jeder Auflage aufs neue willkommen geheissen wird!

Redaktion des Auskunftsbuches für die ohemische Industrie. H. Blücher, Chemiker

Leipzig, Körnerstrasse 37.

\title{
Aus dem Vorwort zum II. Jahrgang.
}

Das "Auskunftsbuch für die chemische Industrie“ soll, wie sein Name besagt, dem Chemiker und Industriellen der einschlägigen Gebiete in den Fragen der Praxis Auskunft erteilen; es ist nicht zum Unterricht bestimmt und nimmt eine Mittelstellung zwischen Lehrbüchern und lexikalischen Werken ein. Die rein alphabetische Anordnung gewährt eine schnelle Übersicht; die kurze prägnante Darstellung, die alles weniger wichtige und theoretische übergeht, vermeidet einen übermässigen Umfang, der die Handlichkeit stören müsste.

Seinem Zwecke entsprechend berücksichtigt das Buch, nach grossen Schlagwortgruppen geordnet, alle für die chemische Industrie sowie für den praktischen Gebrauch im chemischen Laboratorium wichtigeren Stoffe und Produkte, weiter die Materialien zu ihrer Erzeugung, dann die Fabrikationsmaschinen und Apparate, Untersuchungsinstrumente, Gerätschaften u. s.w.

Bei den einzelnen Artikeln sind, soweit tunlich, die Formeln, Atom- bezw. Molekulargewichte, die wichtigen und üblichen Dar- 
stellungsmethoden mit den Reaktionsgleichungen, spez. Gewichte, Schmelz- und Siedepunkte, Lösungskoeffizienten, thermo- und elektrochemische Daten, tabellarische Übersichten u. s. w. verzeichnet alles nach Möglichkeit dem derzeitigen Stande der Technik angepasst.

Mit noch lebhafterem Danke als bei der Bearbeitung des ersten Jahrgangs müssen wir der weitgehenden Unterstützung gedenken, die uns von seiten der chemischen Industrie und der damit in Berührung stehenden Firmen mit wenigen Ausnahmen überall entgegengebracht worden ist und die es uns allein ermöglicht hat, der chemischen Technik mit wirklich zuverlässigen Angaben zu dienen.

Selbstverständlich kann das Buch nicht dem Spezialtechniker Winke für die Fabrikation seiner Artikel geben; das verbietet der Umfang und ist auch nicht der Zweck des Buches. Dagegen soll das Auskunftsbuch die chemische Technik soweit zusammenfassen, dass es dem Nachschlagenden ermöglicht, sich über alle ihm selbst ferner stehenden Spezialgebiete in willkommener Weise zu informieren und das zu rekapitulieren, was sonst dem Chemiker bei der Mannigfaltigkeit der Einzelzweige gar nicht alles gegenwärtig bleiben kann; dies gilt besonders auch von Neuerungen, welche berücksichtigt wurden, soweit sie sich praktisch bewährt haben und soweit sich zuverlässige Angaben darüber erlangen liessen. Die gegebenen tabellarischen Übersichten, Zahlenangaben u. s. w. werden selbstverständlich auch dem Spezialtechniker nützlich erscheinen.

Als praktisch besonders wertvoll dürfte sich die Angabe von Preisen erweisen. Die Preise sind allerdings bel den meisten Artikeln sehr schwankend, doch werden sie mit seltnen Ausnahmen im Verlauf eines Jahres (bis zum Erscheinen des nächstjährigen Auskunftsbuches) wenigstens soweit Gültigkeit behalten, um jedem Interessenten eine rasche Orientierung und eine praktisch genügende ungefähre Kalkulation zu ermöglichen. Freilich liegt eine Ungleichheit der aufgenommenen Preise darin, dass solche teilweise von Fabrikanten, teilweise von Grosshändlern u.s. w. stammen; eine Klärung und Einheitlichkeit in dieser Hinsicht lässt sich erst im Laufe der Jahre erreichen.

Wir hoffen, dass in der Folge auch diejenigen wenigen Firmen, die hinsichtlich Angabe von Preisen bisher zurückhaltend geblieben sind, da sie aus solchen Angaben Vorteile für ihre Konkurrenz fürchteten, uns in der Folge ebenfalls durch Preisstellung zu Danke verpflichten werden, da, wie der Text erweist, alle Preise ohne spezielle Firmennennung aufgeführt sind und eben nur der ungefähren Information des Lesers dienen sollen. 
Wir betonen immer wieder, dass nur die Unterstützung aller Kreise der chemischen Industrie unser Werk $2 \mathfrak{u}$ dem machen kann, was es werden will: $z u$ einem willkommenen Jahrbuch, zu einem zuverlässigen Ratgeber, zu einem vertrauenswürdigen Nachschlagebuch für die Praxis.

Was die unter den einzelnen Artikeln verzeichneten Bezugsquellen und die eingefügten Inserate anlangt, so sei ausdrücklich betont, dass die Einschiebungen von einem $A$ uftrage der betreffenden Firmen beim Verlage abhängen und mit dem Text der Artikel nichts zu tun haben; die Redaktion hat hinsichtlich des Textes überall strengste Objektivität gewahrt. Ein Adressbuch ist das vorliegende Werk nicht; deshalb wäre es unangebracht, unter den Firmen alle Lieferanten zu suchen oder überhaupt die Angabe von Firmen als einen integrierenden Bestandteil des Werkes zu betrachten; aber wir hoffen, dass die Bereitwilligkeit des Verlages, Bezugsquellen mit aufzunehmen, unsern Lesern in vielen Fällen eine wertvolle und willkommene Information hinsichtlich der Lieferanten gewähren wird.

Was den vorliegenden $z$ weiten Jahrgang anlangt, so ist der ganze Inhalt des Buches genau revidiert worden; durch eingehende Berücksichtigung der neuesten Fachliteratur und namentlich der Patentschriften ist es möglich geworden, den Leser mit allen wertvollen Neuerungen bekannt zu machen. So stellt sich der zweite Jahrgang in wesentlich veränderter und verbesserter Gestalt dar. Zahlreiche Artikel sind erheblich erweitert, viele andere ganz neu aufgenommen worden. Als eine der beachtenswertesten Neuerungen dieses Jahrgangs nennen wir, dass, soweit angängig, bei den wichtigeren Artikeln die technischen Prüfungsmethoden eingefügt worden sind. Vorausgesetzt wurde dabei naturgemäss die Beherrschung der allgemeinen analytischen Methoden; teilweise wurde auf die Prüfungsverfahren des Deutschen Arzneibuchs (D. A. IV) verwiesen. Wir glauben, unsern Lesern gerade mit den Prüfungsmethoden eine willkommene Gabe zu bieten. 